\title{
A High-bandwidth PWM Servo Amplifier For The Direct-Drive-Valve Actuation System
}

\author{
Jea-Sen Lin Chern-Lin Chen \\ Dept. of Electrical Engineering \\ National Taiwan University \\ Taipei, Taiwan
}

\begin{abstract}
A high-bandwidth bi-quadrant buck/boost servo amplifier with nonlinear PWM control for the direct-drive-valve flight control actuation system is proposed in this paper. In the original H-bridge amplifier control loop, proper feedback compensation fails to enhance the system bandwidth due to nonlinear saturation effect. The designed function of the presented circuit is to increase the amplifier's slew rate by extending its linear operation range. Experimental results show that the system bandwidth is enlarged from originally $150 \mathrm{~Hz}$ to $500 \mathrm{~Hz}$.
\end{abstract}

\section{INTRODUCTION}

The Direct-Drive-Valve (DDV) actuation system studied in this paper is equipped in the light-weight dual-engine Taiwanese Indigenous Defensive Fighter (IDF) to dynamically manipulate five primary control surfaces and one secondary surface. The DDV hydraulic actuator is a new generation of electro-mechanical actuation system. Its basic schematic concept is shown in Fig. 1.

The performance characteristics of DDV actuation are comparable to the Electro-Hydraulic Valve (EHV)[1],

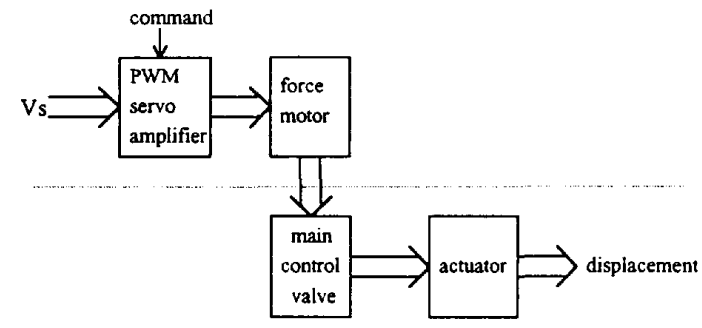

Fig. 1 Basic schematic concept of DDV actuation system

\author{
Chen-Yan Lai \\ Aeronautical Research Lab. \\ Chung-Shan Inst. of Sci. \& Tech. \\ Taichung, Taiwan
}

but offers distinct advantages over the EHV concept. Of significance are the complete elimination of the redundant electro-hydraulic servovalves and the accompanying mechanical failure detection that burden the EHV. The DDV actuation system uses redundant electronic inner-loop feedback control and failure detection of the single main control valve (MCV). This not only simplifies the actuator design, but also provides significant weight savings. The single-stage DDV also allows for high-gain closed-loop operation, offering excellent threshold and dynamic performance. In addition, the single-stage design provides a higher natural frequency of the MCV as well as an overall reduction in hydraulic power consumption[2].

The DDV actuator, however, does have its limitations. Unlike the two-stage EHV actuator, the DDV, with its single-stage valve, requires a much higher current to drive the force motor (FM) coil. Originally, a pulse-width-modulated (PWM) full-bridge servo amplifier[3] is used to drive the FM coil. The bandwidth of the input current to the FM is approximately $150 \mathrm{~Hz}$, due to the high inductance in the FM coil. To speed up the FM's response, the input current bandwidth has to be enlarged. And then the response speed of the whole DDV actuation system can be improved.

\section{BANDWIDTH ANALYSIS OF THE SERVO SYSTEM}

Fig. 2 shows the functional block diagram of the control for the DDV actuation system. With the servo amplifier working in the linear region, $\mathrm{V}_{\mathrm{O}}=\mathrm{K}_{\mathrm{A}} * \mathrm{~V}_{\mathrm{C}}$, and a proportional-integral type controller, $\mathrm{G}_{\mathrm{C}}(\mathrm{s})=\mathrm{K}_{\mathrm{p}}+\mathrm{K}_{\mathrm{I}} / \mathrm{s}$, the transfer function between the input command $I_{\text {com }}$ and the current response $\mathrm{I}_{\mathbf{a}}$ is

$$
\left.\frac{I_{a}(S)}{I_{c o m}(S)}\right|_{F_{m}=0}=\frac{K_{A}\left(K_{P} S+K_{I}\right) / L_{a}}{S^{2}+2 \xi \omega_{n} S+\omega_{n}^{2}}
$$

The function's response indices can be derived as 


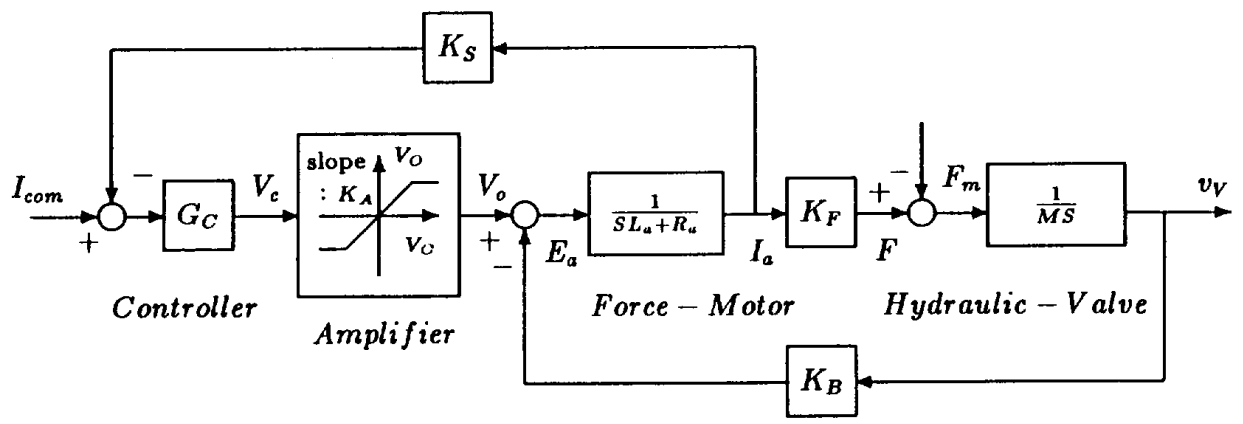

Fig. 2 Functional block diagram of the DDV actuation system

resonant frequency

$$
\omega_{n}=\sqrt{\frac{M K_{L} K_{A} K_{S}+K_{F} K_{B}}{M L_{a}}}
$$

damping ratio

$$
\xi=\frac{\left(R_{a}+K_{A} K_{S} K_{P}\right) \sqrt{M}}{2 \sqrt{L_{a} M K_{I} K_{A} K_{S}+L_{a} K_{F} K_{B}}}
$$

bandwidth

$$
B W=\omega_{n} \sqrt{1-2 \xi^{2}+\sqrt{4 \xi^{4}-4 \xi^{2}+2}}
$$

In the real system, a high feedback gain in the servo amplifier control loop fails to improve the slew rate of the PWM servo amplifier. A high feedback gain increases the control performance, only if the amplifier is operated in its linear amplification region. Following a control command, the error signal is initially too large to stay in the linear region. The amplifier is driven into its saturation region and its output voltage is fixed. The system states are uncontrollable to the control command at this time.

The functional diagram of the DDV actuation system with a saturated servo amplifier is shown in Fig. 3. The value of the saturated output voltage has nothing to do with the feedback gain or the error signal level. It is determined by the characteristics of the servo amplifier. The transfer function between the armature current $L_{\text {and }}$ and the amplifier's output voltage $V_{0}$ is given as

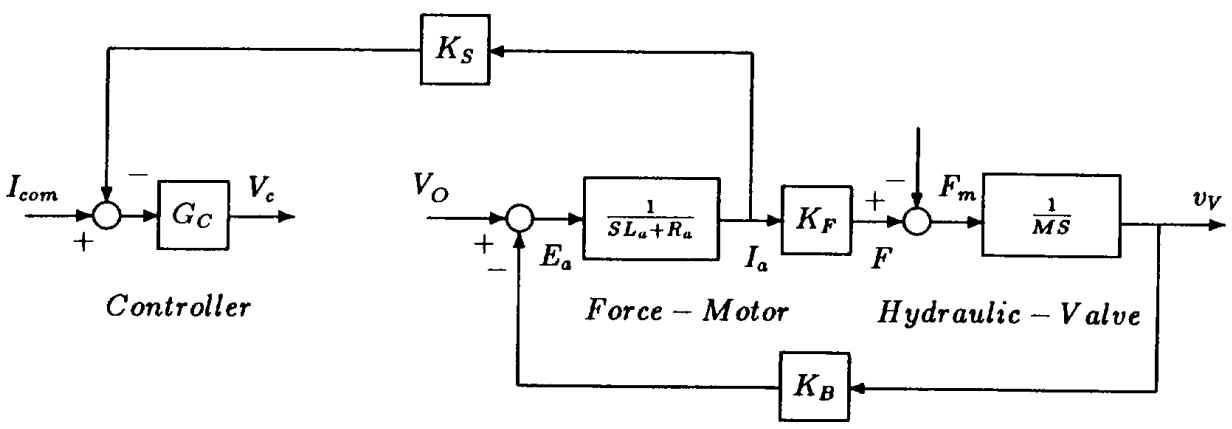

Fig. 3 Functional block diagram of the saturated DDV system 


$$
\left.\frac{I_{a}(S)}{V_{O}(S)}\right|_{F_{m}=0}=\frac{M S}{M L_{a} S^{2}+M R_{a} S+K_{F} K_{B}}
$$

Obviously, the bandwidth of the armature current is not affected by the feedback gain.

\section{THE BI-QUADRANT BUCK/BOOST SERVO AMPLIFIER}

From the above mathematical analysis, the bandwidth of the servo system can be improved with proper feedback only when it works in the linear amplification region. The amplifier's output saturation voltage determines the size of this linear section. Yet, the maximum output voltage of a conventional full-bridge servo amplifier is bounded by its input voltage, which is usually 28 volts in aeroplanes. In order to improve the response of the DDV actuation system, a bi-quadrant buck/boost converter is designed to replace the full-bridge amplifier. Fig. 4 gives the proposed power circuit. It combines a full-bridge with a buck/boost converter and has the advantages of both. Its output voltage can be boosted and can be reversed, depending on actuation requirements.

The front end is a full-bridge converter consists of Q1, Q2, Q3, and Q4. The direction of the input current is restricted by $\mathrm{D} 1$ and $\mathrm{D} 2$ to avoid unwanted inductor discharging. This full bridge controls the input voltage polarity and the on/off duty cycle for charging the energy-storing inductor L. Q5 and Q6 form a controllable bi-directional switch which takes the role of the output rectifying diode in a buck/boost converter. The back-to-back orientations prevent false conduction of their intrinsic body diodes. The output filter capacitor $\mathrm{C}$ has to be

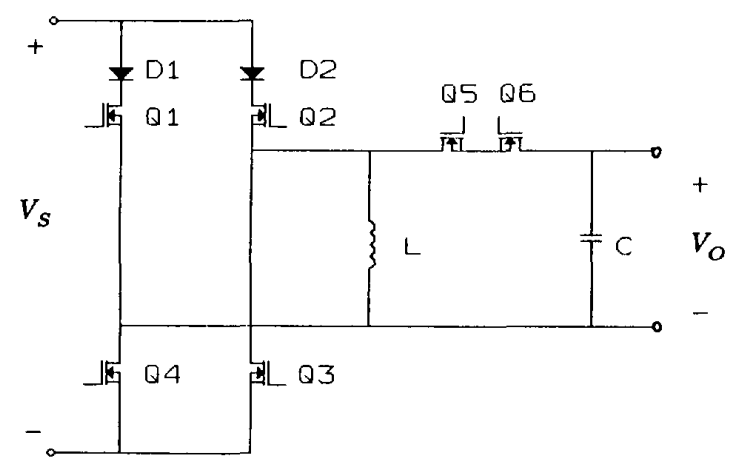

Fig. 4 Configuration of the proposed servo amplifier bi-directional in order to provide positive and negative output voltages. In this way, the proposed servo amplifier can work in the first and third quandrants in the plane of its output voltage and current.

Using the state-space averaging approach [4] to model the proposed circuit, the output voltage can be represented by a nonlinear function of the duty cycle. Fig. 5 gives the output voltage response versus duty ratio of the designed circuit. Two cases are studied, one with $10 \Omega$ load resistance and the other with $100 \Omega .120 \mu \mathrm{H}$ inductor with $0.65 \Omega$ series resistance and $12 \mu \mathrm{F}$ bi-directional capacitors with $0.05 \Omega$ equivalent series resistance (ESR) are used. In this figure, the simulated results are shown by solid curves and the experimental results by dots of ' $o$ ' $\&$ ' $x$ '. The experimental values are only slightly less than simulated.

\section{NONLINEAR PWM CONTROL}

The control characteristic of the bi-quadrant buck/boost converter is nonlinear, that is, its incremental gain varies as the operating point moves. This is an undesirable feature for a system, if we want to close a feedback loop around it. To make its gain constant over the entire operating range, the control characteristic is linearized using a new feedback design technique.

An exponential function approximating the inverse function of the nonlinear control characteristic is used as the carrier for generating PWM control signals, as shown in Fig. 6. The nonlinear modulation mechanism compensates for the nonlinear control characteristic and then gives nearly constant incremental gain of the feedback loop over the entire operating range. Fig. 7 depicts this new design concept.

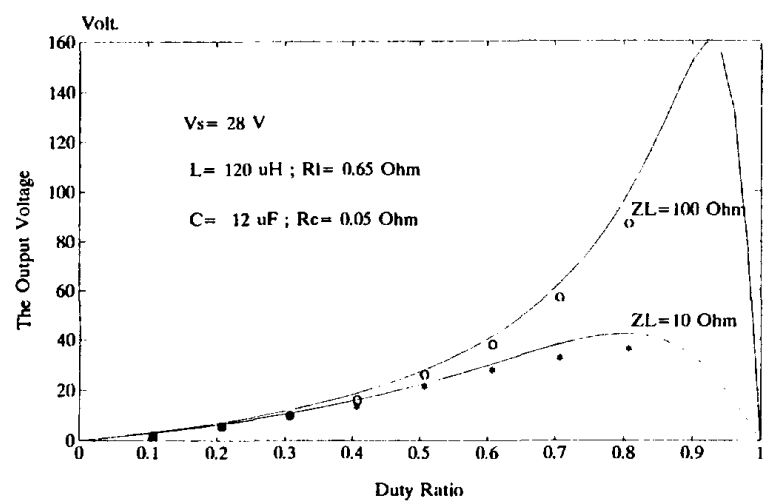

Fig. 5 Output voltage response of the designed circuit 


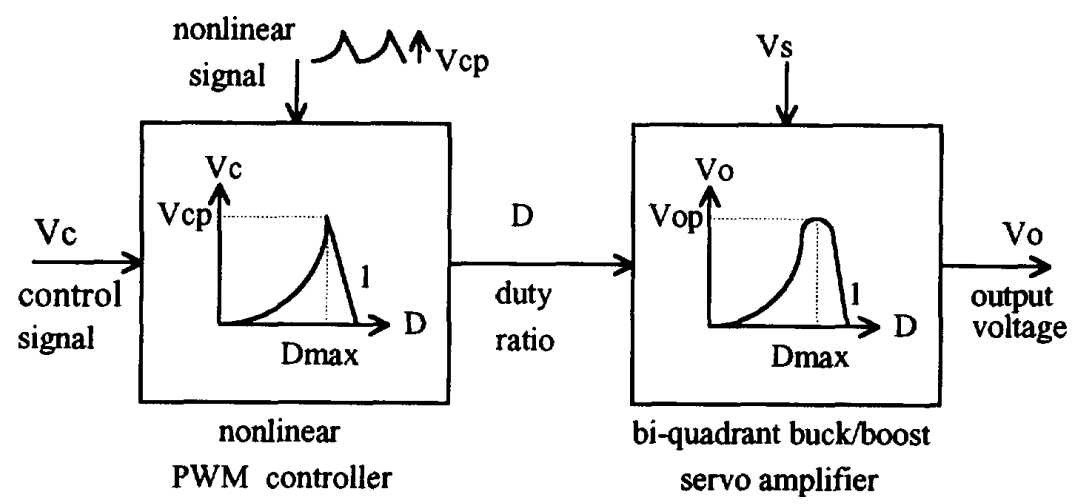

Fig. 6 PWM control with an exponential modulating signal

\section{EXPERIMENTAL IMPLEMENTATION AND RESULTS}

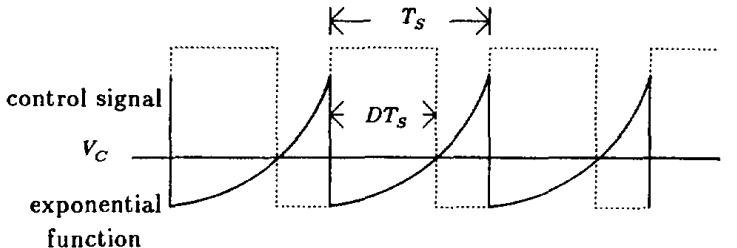

Fig. 7 Nonlinear PWM control scheme
The complete system of the implemented servo amplifier include six major parts: the bi-quadrant buck/boost power circuit, PWM control circuit, gate drive circuit, feedback compensation, signal procesing circuit, and auxiliary power supply. Fig. 8 shows the functional blocks of this system. Experiments are performed on a $100 \mathrm{mH}$ dummy load to simulate the high inductance in the FM. The normal value is above $70 \mathrm{mH}$. This high inductance is the cause of the FM's slow input current response. Low bandwidth, about $150 \mathrm{~Hz}$, is observed in the original system drived by a full-bridge servo amplifier. As for the designed bi-quadrant buck/boost amplifier, Fig. 9 depicts that

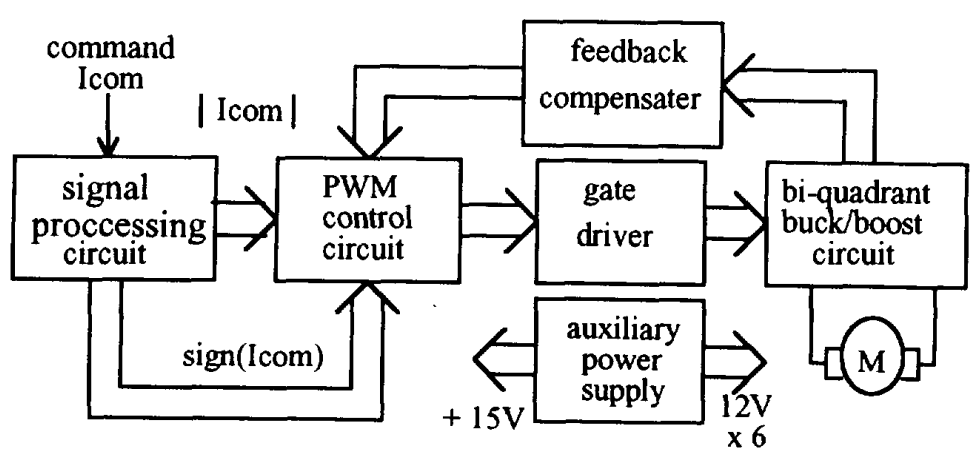

Fig. 8 Functional blocks of the implemented circuit 
following the control command the amplifier's output current rapidly grows. The bandwidth is enlarged to about $500 \mathrm{~Hz}$, as shown in Fig. 10.

\section{CONCLUSIONS}

In this study, a bi-quadrant buck/boost PWM DC servo amplifier for the DDV flight control actuation system is presented. The design feature that contributes most to the high bandwidth of the servo amplifier is the output voltage boost and reversal. A nonlinear PWM control with an exponential modulating signal is also proposed to compensate the nonlinear control characteristics of the power circuit. Experiments show that the bandwidth can be enlarged from $150 \mathrm{~Hz}$ to $500 \mathrm{~Hz}$. Though this design originally aims at aeroplane actuation, it is the authors' belief that it can also be used for other high-bandwidth DC drives where fast servo responses are required.

\section{REFERENCES}

[1] H. E. Merritt, Hydraulic Control Systems, Chap. 7, John Wiley \& Sons, 1967.

[2] Y. N. Hsu, C. Y. Lai, M. H. Hsu, Y. K. Lee, "Development of the direct drive valve (DDV) actuation system on the IDF aircraft," International Pacific Air \& Space Technology Conference and 29th Aircraft Syposium, Japan, Oct. 1991.

[3] N. Mohan, T. M. Undeland, W. P. Robbins, Power Electronics : Converters, Applications, and Design, Chap. 5, John Wiley \& Sons, 1989.

[4] R. D. Middlebrook, "Small-signal modeling of pulse-width modulated switched-mode power converters," IEEE Proc., Vol. 76, pp.343-354, 1988.

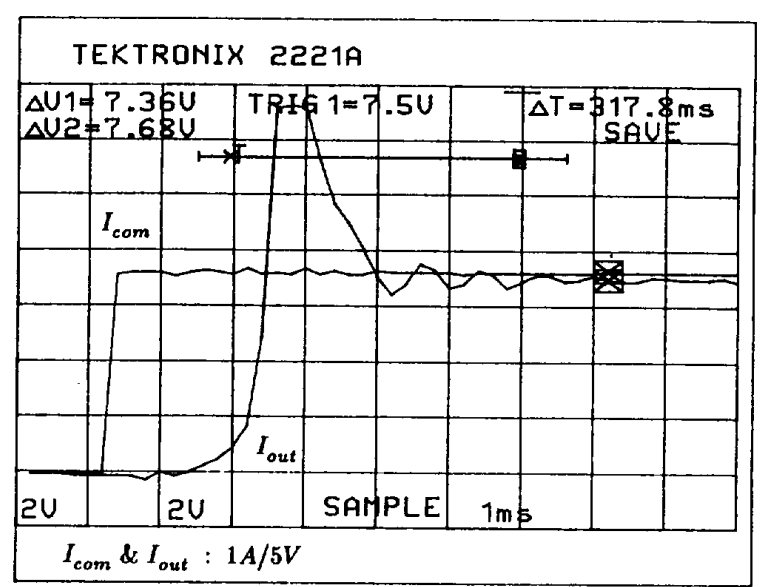

Fig. 9 Armature current response following a control command
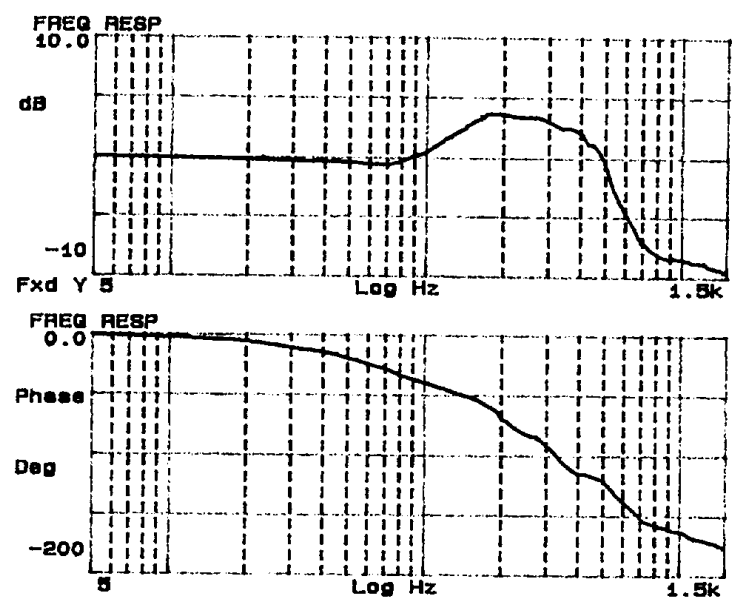

Fig. 10 Frequency response of armature current 